\title{
Imaging in Liquids through Ultra-thin Membranes: A Comparative Analysis of Scanning Electron and Scanning Microwave Microscopies
}

\author{
Alexander Tselev ${ }^{1,2}$, Jeyavel Velmurugan ${ }^{3,4}$ and Andrei Kolmakov ${ }^{3 *}$ \\ ${ }^{1}$ Center for Nanophase Materials Sciences, Oak Ridge National Laboratory, Oak Ridge, TN 37831, USA \\ ${ }^{2}$ Institute for Functional Imaging of Materials, Oak Ridge National Laboratory, Oak Ridge, TN 37831 , \\ USA \\ ${ }^{3}$ Center for Nanoscale Science and Technology, National Institute of Standards and Technology, \\ Gaithersburg, MD 20899 \\ ${ }^{4}$ Maryland Nano Center, University of Maryland, College Park, MD 20742
}

The ability of the near-field microwave microscopy to image subsurface structures has been realized shortly after the invention of the technique [1-3]. Based on these capabilities, we proposed and successfully tested a novel concept of in situ near-field scanning microwave impedance microscopy (sMIM) for imaging of reactive and biological samples [4, 5]. In our approach, the samples of interest were separated from ambient by a few nanometer thick dielectric membranes transparent for microwave radiation. Different from prior examples of microwave imaging in liquids, where the probe had to be immersed in a liquid or exposed to aggressive vapors [6,7], our approach benefits from complete isolation of the probe and the rest of the microscope from reactive environment and therefore enables in situ imaging of previously inaccessible highly reactive, toxic, corrosive, or radioactive samples. Here we report on a comparative side-by-side study of imaging capabilities of liquid scanning electron microscopy (SEM) and liquid sMIM techniques using the same set of biological and inorganic samples. In particular, the sensitivity, spatial resolution, probing depth, scanning rate, and probe induced effects are compared.

Figure 1 demonstrates, the experimental setup and nanoscale images of electrochemical processes in liquids, using microwave near-field, formed at a sharp scanning probe. In particular, we demonstrate in situ real time imaging of metal $(\mathrm{Ag})$ dendrites growth at electrode-liquid electrolyte interface during electrochemical plating reaction. The panel (d) shows low resolution SEM image of the same sample area as in (c). The contrast of the dendrites in secondary electron images confirms their higher $\mathrm{Z}$ number compared to the liquid electrolyte. The central part depicts the attempt to acquire higher resolution SEM image. The degradation of Ag deposit is due to electron beam induced oxidation of metal $\mathrm{Ag}$ and its dissolution by the electrolyte [8],[9]. Different to the liquid SEM and transmission electron microscopy, sMIM imaging is nondestructive, free from beam-induced artifacts and can be readily applied to electrochemical studies thanks to the extremely low energy deposited to the sample. Indeed, the energy of $3 \mathrm{GHz}$ microwaves is of the order of $\approx 10 \mu \mathrm{eV}$, which can affect neither electronic states nor chemical bonds in condensed matter.

The demonstrated spatial resolution of sMIM imaging in our experiment was $\approx 250 \mathrm{~nm}$. However, the resolution can be improved since it depends on parameters such as probe tip radius, membrane thickness and dielectric constants difference. Under optimal conditions, a resolution of $\approx 50 \mathrm{~nm}$ can be achieved for metallic objects with the commercially available probes with $\approx 50 \mathrm{~nm}$ tip apex radius. Such resolution is comparable to that demonstrated by SEM in liquids [10]. 
References:

[1] K. Lai, et al. Review of Scientific Instruments, vol. 78, 2007, p. 063702.

[2] C. Plassard, et al., Physical Review B, vol. 83, 2011, p. 121409.

[3] J. J. Kopanski, et al. ECS Transactions, vol. 61, 2014, pp. 185-193.

[4] A. Tselev and A. Kolmakov, Microwave Conference (EuMC), 2015 European, 2015, pp. 662-665.

[5] A. Tselev, et al. vol. 10 (3), 2016, pp 3562-3570.

[6] S. Kim, et al, Applied Physics Letters, vol. 86, 2005, p. 153506.

[7] M. Farina, et al, Microwave and Wireless Components Letters, IEEE, vol. 22, 2012, pp. 595-597.

[8] K. W. Noh, et al, Ultramicroscopy, vol. 116, 2012, pp. 34-38.

[9] N. M. Schneider, et al, The Journal of Physical Chemistry C, vol. 118, 2014, pp. 22373-22382.

[10] S. Thiberge, et al, Review of Scientific Instruments, vol. 75, 2004, pp. 2280-2289.

[11] The research was supported in part through Scientific User Facilities Division (ORNL), Office of Basic Energy Sciences, U. S. Department of Energy, U.S. Civilian Research and Development

Foundation and NIST-CNST/UMD-IREAP Cooperative Agreement
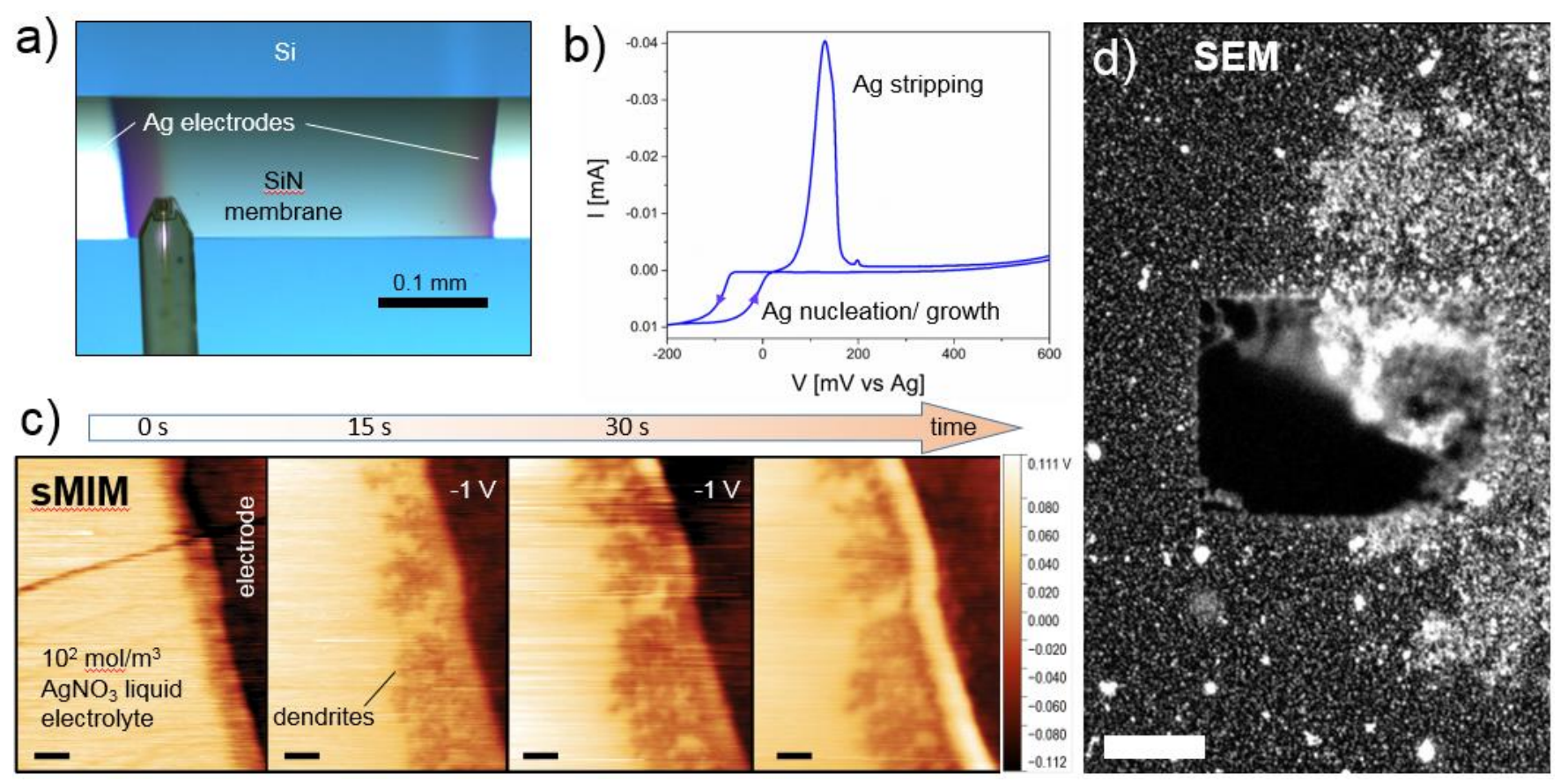

Figure 1. (a) Optical bright field top view of the electrochemical environmental cell for sMIM imaging in liquid electrolytes with sMIM probe scanning over the SiN membrane. (b) A cyclic voltammogram of $\mathrm{Ag}$ dendritic growth and stripping at/from Ag electrode of the environmental cell. (c) sMIM-C maps of electrochemical Ag dendritic growth on Ag cathode taken at open circuit condition induced by a sequence of the applied potential (-1 V, $15 \mathrm{~s}$ ) steps. (d) Comparative liquid SEM image of the same dendrites. An attempt to acquire high-resolution SEM image of the dendrite apex leads to beam induced dendrite oxidation and dissolution by electrolyte. Scale bars in (b) and (d) are $100 \mu \mathrm{m}$ and $2 \mu \mathrm{m}$ correspondingly. 